\title{
Top-Down Coarse-Grained Framework for Characterizing Mixed Conducting Polymers
}

\author{
Aditi Khot and Brett M. Savoie* \\ Davidson School of Chemical Engineering, Purdue University, West Lafayette, IN, 47906 \\ E-mail: bsavoie@purdue.edu \\ Phone: 765-494-4235
}

\begin{abstract}
Organic polymers that exhibit both ionic and electronic conduction are of interest for energy storage devices and emerging bioelectronic applications. Nevertheless, organic mixed conductors are at an early stage of development with nascent design rules and relatively few material chemistries having been experimentally characterized. Here we report a coarse-grained modeling framework that is sufficiently flexible to represent a range of mixed conducting chemistries while retaining the molecular physics necessary to interrogate structure-function relationships. A detailed overview of the framework is presented, accompanied by an applied study of the effect of hydration and oxidation levels on a representative mixed conductor. The model recapitulates experimental trends related to the macroscopic ionic and electronic conductivities, including the non-linear suppression of the electronic mobility with respect to oxidation level and the direct relationship between ionic mobility and hydration level, while revealing the complex interplay of polymer morphology, ionic-electronic coupling, and electrolyte distribution that govern these relationships. These results provide a validation of this framework for future applications in establishing structure-function relationships in this important materials class, and suggests several near-term opportunities for tailoring mixed conduction via side-chain design.
\end{abstract}

\section{Introduction}

Organic mixed ionic electronic conductors ${ }^{1}$ (OMIECs) are an ascendant class of organic electronic materials that exhibit both ionic and electronic transport. The ability to transport both ions and polarons in a single material is useful in energy storage ${ }^{2-4}$ (e.g., as battery electrodes components) and sensing applications ${ }^{5-8}$ (e.g., active layers in organic electrochemical transistors), where ion-mediated redox processes within the OMIEC are macroscopically coupled to an external circuit via the electronic conductivity. In these applications, ion-mediated doping occurs volumetrically within the OMIEC, via an electrolyte phase that co-percolates with an electronic conducting phase throughout the volume of the material. This is a defining feature of OMIECs that drives the need for distinct design rules and structure-function re- lationships as compared to those that have been separately established for organic semiconductors and organic electrolytes (i.e., organic materials that conduct electrons, or ions, but not both).

Co-percolation of electronic and conducting phases within OMIECs also presents several fundamental mechanistic questions that have yet to be generally addressed. ${ }^{7,9}$ First, these phases are coupled both by their intimate mixing (typically $<100$ $\mathrm{nm}$ ) and through significant morphology changes that accompany the (de)doping processes. ${ }^{10-13} \mathrm{Al}$ though it is documented that morphology changes can impact material stability ${ }^{14-16}$ and transport coefficients by many orders of magnitude, ${ }^{10,17}$ the extent to which morphologies can be tailored, or what constitutes an optimal morphology in the context of OMIECs is still unknown. ${ }^{18-20}$ Second, the presence of mobile ions and polarons in the same material substantially alters the descrip- 
tion of charge transport relative to typical organic semiconductors and conductors. ${ }^{21-25}$ On the one hand, potentially strong electrostatic interactions between ions and polarons presents the possibility of significant charge trapping and ion-coupled electron transport. On the other hand, depending on the charge densities and characteristics of the electrolyte phase (e.g., aqueous vs. organic) screening may mitigate trapping. ${ }^{10}$ At present, these mechanistic details are difficult to interrogate experimentally, given the short lengthscales involved, the required temporal resolution, and the disordered nature of these soft materials.

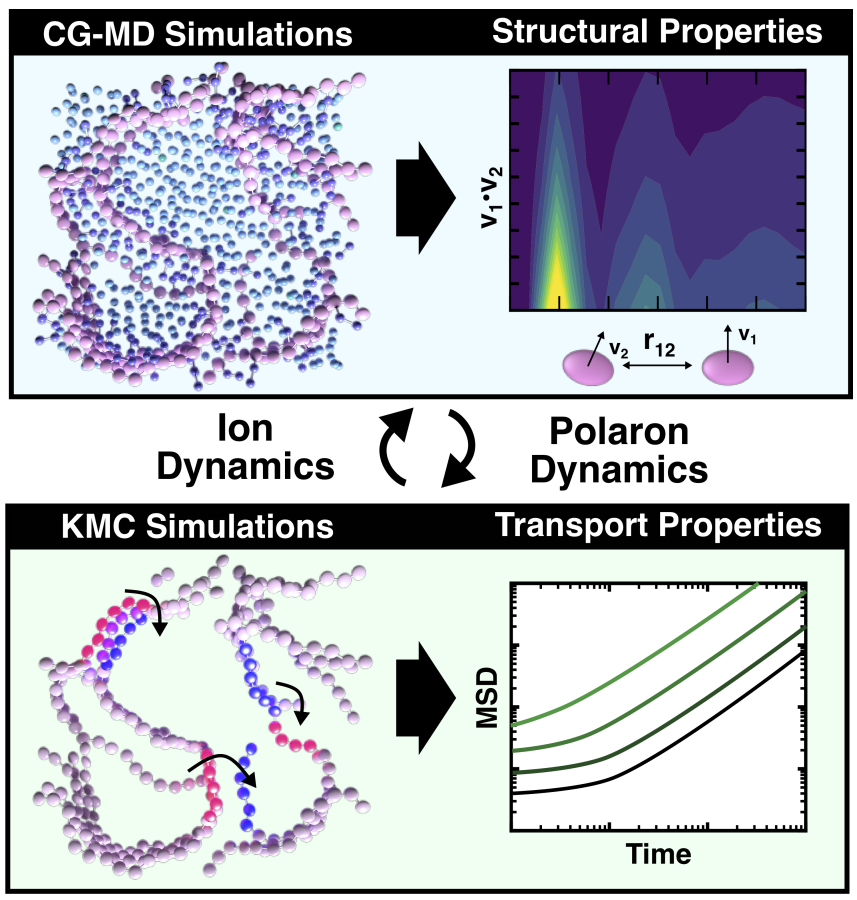

Figure 1: Overview of the simulation methodology developed in this work. (Top) Coarse-grained molecular dynamics simulations with anisotropic polymer backbone interactions are used to described mixed conductor morphology. (Bottom) Kinetic Monte Carlo simulations based on configurationally averaged rates are used to characterize polaron dynamics.

To address these aspects of OMIEC operation, we have developed a generic coarsegrained molecular dynamics (CG-MD) model of OMIECs and coupled it with a kinetic Monte Carlo (KMC) ${ }^{26}$ model of charge transport (Fig. 1). This modeling framework provides a description of both electronic and ionic transport mechanisms at lengthscales of 10-100 nm, which fills a critical characterization gap in contemporary experimental approaches. The KMC methodology that we develop builds on a relatively mature approach to modeling charge transport in organic semiconductors, but with extensions to account for the distinct ion-electronic coupling mechanisms in OMIECs. These KMC simulations utilize CG-MD configurations with a mean-field treatment of electrons and an explicit description of the electrolyte and polymer nuclear degrees of freedom. At present, only one CG-MD model of OMIECs has been published specific to the mixed conducting polymer PEDOT. ${ }^{27-31}$ Thus, we have also developed a generic CG-MD force-field for OMIECs that can be used to systematically interrogate the various designable OMIEC components and their effect on morphology and transport processes. As an initial benchmark demonstration of this framework, we have investigated effects of oxidation and hydration levels on the morphology and transport processes of a generic p-type conjugated homopolymer with glycolated sidechains in the presence of an aqueous electrolyte.

\section{Methods}

\subsection{Mixed Conduction Model}

An overview of the mixed conduction workflow is presented in Fig. 1. The details of each step are described in the subsequent sections but are briefly discussed here to guide the reader. The first step is the simulation of mixed conductor configurations at the coarse-grained level for a given polymer and electrolyte. For this purpose, we have developed a general coarse-grained model that can represent a broad class of OMIEC chemistries (Section 2.2). Molecular dynamics is used to characterize the equilibrium behavior of the mixed conductor and ionic diffusivity at different conditions (Sections 2.3 and 2.4). Based on these configurations, polaron transport is modeled as hopping between localized sites along the conjugated polymer backbones. The rates for hopping are determined by semi-classical Marcus theory in a manner that incorporates the details of charge delocalization, intrachain vs. interchain hopping, and local electrostatic contributions to the site energies (Section 
2.5). The resulting set of polaron transport processes forms a master equation that we evaluate using the Kinetic Monte Carlo algorithm (Section 2.6). This approach yields average polaron mobilities, ionic mobilities, and scattering functions that can be compared with macroscopic measurements, as well as the distribution of transport pathways, their relative resistance, and microscopic ion-polymer configurations that are currently inaccessible by experiments.

\subsection{Coarse-Grained Molecular Dy- namics Model}

(a)
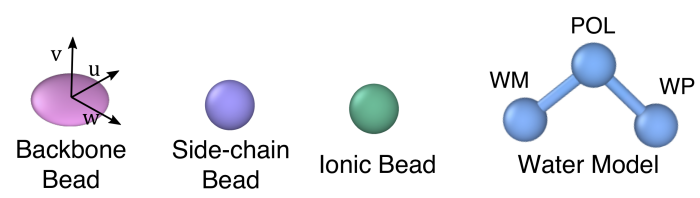

(b)

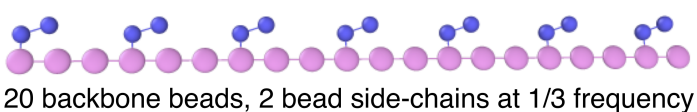

Figure 2: Overview of the distinct bead-types used here for coarse-grained simulations of mixed conductors: (a) Building blocks of the generic CG model. For polymers with more complex sequence or side-chain structure, multiple beads might be utilized in each category. (b) The polymer structure used in the present study.

The essential features of a mixed conducting, conjugated polymer electrolyte system consist of four components-conjugated backbone, polar side-chain, ions, and solvent (Fig. 2). We have developed a flexible top-down framework for modeling each component that can be used to represent a broad range of chemistries relevant to OMIECs. The general features of the model are described here, while parameterization details are described in the following section.

To reproduce the anisotropy of the $\pi$-system, the conjugated beads are treated as ellipsoids. The Gay-Berne potential ${ }^{32}$ is used to model anisotropic interactions between ellipsoid particles with radii $\sigma_{u}, \sigma_{v}$ and $\sigma_{w}$ and potential minima $\varepsilon_{u}, \varepsilon_{v}$ and $\varepsilon_{w}$, in the directions $\mathbf{u}, \mathbf{v}$ and $\mathbf{w}$, respectively (Fig. 2a). The Gay-Berne potential is derived by treating these ellipsoids as gaussian volumes, evaluating their overlap integral and mapping the integral on to a Lennard-Jones type potential with some empirical modifications, resulting in the expression

$$
\begin{array}{r}
U_{12}\left(\omega_{1}, \omega_{2}, \mathbf{r}_{12}\right)= \\
4 \varepsilon_{0} \varepsilon_{12}^{v}\left(\omega_{1}, \omega_{2}\right) \varepsilon_{12}^{\prime \mu}\left(\omega_{1}, \omega_{2}, \hat{\mathbf{r}}_{12}\right) \times \\
{\left[\left(\frac{\sigma_{c}}{r_{12}-\sigma_{12}\left(\omega_{1}, \omega_{2}, \hat{\mathbf{r}}_{12}\right)+\sigma_{c}}\right)^{12}\right.} \\
\left.-\left(\frac{\sigma_{c}}{r_{12}-\sigma_{12}\left(\omega_{1}, \omega_{2}, \hat{\mathbf{r}}_{12}\right)+\sigma_{c}}\right)^{6}\right],
\end{array}
$$

where the orientation of the particles is given by their Euler angles, $\omega_{1}$ and $\omega_{2}$, and $\mathbf{r}_{12}$ is the displacement vector between ellipsoids. In Eq. 1, $\varepsilon_{12}$ and $\varepsilon_{12}^{\prime}$ are energy prefactors, and $\sigma_{12}$ is the contact radius. The exponents $v$ and $\mu$ are empirical coefficients and $\sigma_{c}$ is the minimum contact radius, introduced to control the width of potential well and avoid unphysical overlaps.

In the current work, the conjugated backbone is modeled by uniaxial ellipsoids with the major axes (equivalent $\mathbf{u}, \mathbf{w}$ ) in the plane of backbone and the minor axis ( $\mathbf{v})$ perpendicular to the backbone (Fig. 2a). The parameters $\varepsilon_{v}$ and $\varepsilon_{u, w}$ govern the interaction minima between conjugated beads along the minor and major axes, respectively. These can be tuned to interrogate OMIECs with variable $\pi$ $\pi$ interactions. The radius $\sigma_{v}$ controls the $\pi-\pi$ stacking distance and the radii $\sigma_{u, w}$ approximate the conjugated ring radius. The non-bonded interactions of all other components of the system (i.e., side-chains, solvent, and ions) are modeled with isotropic Lennard-Jones potentials based on the Martini force-field. The cross-interactions between the backbone beads and the side-chain, solvent and ions are also modeled as isotropic.

Three types of bonded potentials are used to control the orientation of the conjugated backbone beads with respect to their neighbors. The first potential governs the dihedral rotation about the bond connecting backbone beads (r) with respect to the orientation vectors of each bead ( $\mathbf{v})$, as illustrated in Fig. 3a. ${ }^{33}$ This dihedral is modeled using the standard OPLS form and is the analogue of the inter-ring torsions in conjugated systems that dictate the delocalization of polarons along the backbone. ${ }^{34}$ Two additional harmonic poten- 


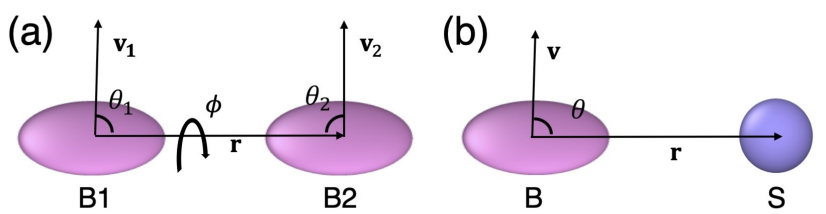

Figure 3: Illustration of the bonded interactions governing the orientation of backbone beads. (a) Dihedral potential between two backbone beads based on their orientation vectors (v). (b) Bending potential between the backbone bead normal and the sidechain bead.

tials are used to model the bending interaction between bonded backbone beads and between backbone beads and bonded sidechain beads, respectively. In each case, the potential is based on the angle between the bond vector, $\mathbf{r}$, and the orientation vector, $\mathbf{v}$, of the backbone bead (Fig. 3ab). The force constant for the backbone-backbone bending term can be modified to vary the stiffness of the backbone. Likewise, the equilibrium angle of the backbone-sidechain bending term can be modified to model sidechains bonded to $s p^{3}$ vs $s p^{2}$ positions along the backbone. The derivation of the forces and torques associated with these potentials can be found in the SI.

The remaining three components, namely, the polymer side-chain, ions, and solvent are modeled as isotropic beads using the Martini force-field. ${ }^{35}$ The Martini parameters have been developed to reproduce experimental partition coefficients for biomolecules and organic molecules using a relatively small number of bead types that typically represent four heavy atoms. Martini bead classification is based on polarity, making it straightforward to vary the polarity of side-chains and solvent, which alters the penetration of solvent and ultimately the transport properties. Each Martini bead is modeled as a Lennard-Jones particle with harmonic bonded interactions and cosine bending terms, where applicable.

\subsection{Parameterization}

The CG framework described in the previous section is capable of modeling a broad range of conjugated polymer electrolyte systems through the appropriate choice of Martini bead-types, and at the same time, captures essential features of $\pi$ - conjugated polymers including anisotropic backbone interactions, controllable planarity, and stiffness. As a baseline benchmark of how this model performs, we have implemented a parameterization representating a p-type conjugated homopolymer with polar sidechains in the presence of an aqueous electrolyte (Fig. 2b). A single backbone bead type was utilized with $\sigma_{u, z}$ and $\sigma_{v}$ parameters chosen to yield repulsive core dimensions of $5 \AA$ along the major axes and $3 \AA$ along the minor axis of the ellipsoid, respectively. To obtain an anisotropy in binding energy of $\sim 5$, which is typical of conjugated polymers, ${ }^{36} \varepsilon_{v}$ was chosen to yield a $1.2 \mathrm{kcal} / \mathrm{mol}$ minimum energy in the $\pi$ stacked orientation and $\varepsilon_{u, w}$ of $0.25 \mathrm{kcal} / \mathrm{mol} \mathrm{mini-}$ mum in side-by-side orientation, similar to coarsegrained Gay-Berne model developed for sexithiophene. ${ }^{37}$ These non-bonded parameters result in backbone beads with dimensions and interaction energies comparable to typical aromatic repeat units. An equilibrium angle of $180^{\circ}$ and force constant of $7.5 \mathrm{kcal} / \mathrm{mol}$ were used for the bending term associated with bonded backbone beads, which results in a persistence length between 4$60 \AA$ for the range of hydration and oxidation levels simulated here (Table S3 \& S4). The backbone dihedral term was chosen to exhibit two minima at $0^{\circ}$ and $180^{\circ}$ with an interconversion barrier of $7.5 \mathrm{kcal} / \mathrm{mol}$, which results in dihedral angle distributions with a standard deviation of $\sim 4.63$ $5.18^{\circ}$ in the simulations. Side-chains composed of two Martini beads ( $~ 8$ heavy atoms in an atomistic representation) were included on every third backbone bead (Fig. 2b). The side-chain bead of type "P1" was used to approximate the CG forcefields developed for conventional OMIEC polyethylene glycol (PEG) side-chains. ${ }^{38}$ An equilibrium angle of $90^{\circ}$ and force constant of $3.0 \mathrm{kcal} / \mathrm{mol}$ were used for the backbone-sidechain bending term. The equilibrium angle of $90^{\circ}$ is meant to mimic a sidechain attached to an $s p^{2}$ backbone atom. The equilibrium morphologies of this system were interrogated as a function of hydration level and oxidation level, then subsequently utilized in charge transport studies. ${ }^{27,39}$ These simulations consisted of 50 chains with 20 repeat units. Oxidation levels of $100 \%, 50 \%, 20 \%, 10 \%$, and $5 \%$, were simulated by equally distributing a positive charge of $1,0.5,0.2,0.1$ and $0.05 q$, respectively, along the 
polymer backbone. ${ }^{38}$ In each case, counter-ions with a unit negative charge and Martini type "Qa" were introduced to maintain charge neutrality. Water is used as the solvent and represented by the Martini polarizable water model. ${ }^{35} \mathrm{~A}$ list of all Martini bead types utilized here and their associated parameters are supplied in Table S2.

\subsection{Molecular Dynamics}

LAMMPS was used to perform all molecular dynamics simulations. ${ }^{40}$ All simulations used a $10 \mathrm{fs}$ integration time step, Velocity-Verlet integration, and periodic boundary conditions. Long-range electrostatics were modeled using the particleparticle-particle-mesh/stagger (PPPM/stagger) algorithm. ${ }^{41-43}$ Long-range electrostatics, LennardJones interactions and Gay-Berne interactions were truncated at $12 \AA$. All simulations were initialized from diffuse configurations, using a cubic grid to place molecules in random orientations without overlap. The velocities were initialized from a uniform distribution obtained with a random seed value and scaled to give the correct kinetic energy. The simulations were first relaxed in the NVE ensemble with restrained displacements of $0.1 \AA$ per time step for 10 ps. This was followed by a simulation in the NVT ensemble at $300 \mathrm{~K}$ while linearly rescaling the simulation box over 2 ns to obtain a condensed system with a density of $1 \mathrm{~g} / \mathrm{cc}$. The density was then equilibrated in the NPT ensemble for $100 \mathrm{~ns}$ at $300 \mathrm{~K}$ and $1 \mathrm{~atm}$. After the NPT simulations, the system was further equilibrated at the average NPT density in the NVT ensemble at $300 \mathrm{~K}$ for $100 \mathrm{~ns}$, followed by a 100 ns production run in the NPT ensemble at 300 $\mathrm{K}$ and $1 \mathrm{~atm}$. These simulation times were chosen to ensure that the polymers diffused 2-3 times its radius of gyration, on average, during the equilibration and production periods, respectively. In both NVT and NPT simulations, the Nosé-Hoover thermostat and barostat were employed using the modified form proposed by Martyna, Tobias, and Klein, as implemented in LAMMPS, ${ }^{44}$ with a relaxation time constant of $0.15 \mathrm{ps}$ and $1.5 \mathrm{ps}$ for the thermostat and barostat, respectively. In all the simulations, the thermodynamic data and coordinates were sampled at $1 \mathrm{ps}$. The ASPHERE package in LAMMPS was used for all Gay-Berne force and torque evaluations and numerical integrations. ${ }^{45}$ The SHAKE algorithm ${ }^{46}$ implemented as a part of the RIGID package in LAMMPS was used to constrain the bonds in the water molecules. The additional bonded potentials to control the orientation of neighboring beads were implemented as custom classes in LAMMPS by our group.

All simulations contained 100 polymers, with 20 backbone beads each. Depending on the backbone bead oxidation level, the number of anionic beads in the simulation box varied to ensure zero net charge on the system. For the oxidation level studies with backbone bead charges of 1.0, 0.5, 0.2, 0.1 , and 0.05 , the total number of anionic beads in the system were 2000, 1000, 400, 200, and 100, respectively. All the oxidation level studies were carried out at $50 \mathrm{wt} \%$ hydration level with 3400 Martini water molecules (i.e., 13600 actual water molecules). For the hydration level studies, the backbone bead oxidation level was fixed at the intermediate value of 0.2 and the simulation box contained 400 anionic beads. The hydration level studies were carried out at $20,35,50,65$, and 80 $\mathrm{wt} / \mathrm{wt} \%$ hydration with $850,1831,3400,6134$, and 13600 water molecules.

\subsection{Charge Transfer Rate Parameters}

Charge transport was modeled here on the basis of configurations generated from the CGMD trajectories. In our model, each backbone bead is parameterized to represent a single conjugated unit (i.e., a ring system without internal flexible dihedral degrees of freedom), thus sites consisting of one or more contiguous backbone beads constitute the relevant polaronic basis states for charge transfer. The charge transfer rates between sites were parameterized using semi-classical Marcus theory, ${ }^{47}$ along similar lines to what has been described in prior work in the context of conjugated polymers. ${ }^{48-51}$ The rate for charge transfer between sites on different chains was modeled in the weak coupling limit as

$$
\omega_{\mathrm{ij}}=\frac{2 \pi}{\hbar} \frac{J_{\mathrm{ij}}^{2}}{\sqrt{4 \pi \lambda_{\mathrm{ij}} k_{B} T}} \exp \left\{-\frac{\left(\Delta E_{\mathrm{ij}}-\lambda_{\mathrm{ij}}\right)^{2}}{4 \pi \lambda_{\mathrm{ij}} k_{B} T}\right\},
$$

where $J_{\mathrm{ij}}$ is the electronic coupling between the sites, $\lambda_{\mathrm{ij}}$ is the reorganization energy, $\Delta E_{\mathrm{ij}}$ is the 
energy difference for a charge occupying the site $\mathrm{j}$ vs site i, $T$ is the system temperature, and $k_{B}$ is the Boltzmann constant. The radial dependence of $J_{\mathrm{ij}}$ was modeled as exponentially decaying with distance as

$$
J_{\mathrm{ij}}=J_{\text {inter }} \exp \left\{-\alpha\left(r_{\mathrm{ij}}-r_{0}\right)\right\}
$$

where $\alpha$ is the decay factor, $r_{0}$ is the reference separation for $J_{\text {inter }}$, and $r_{\mathrm{ij}}$ is the inter-site distance. ${ }^{52}$ The inter-site distance was calculated as an average over the nearest-separation for the beads in each site according to

$r_{\mathrm{ij}}=\frac{1}{2}\left(\frac{\sum_{\mathrm{m} \in \mathrm{i}} \min _{\mathrm{n} \in \mathrm{j}}\left(r_{\mathrm{mn}}\right)}{l_{\mathrm{i}}}+\frac{\sum_{\mathrm{n} \in \mathrm{j}} \min _{\mathrm{m} \in \mathrm{i}}\left(r_{\mathrm{nm}}\right)}{l_{\mathrm{j}}}\right)$,

where the indices $m$ and $n$ run over the beads in sites $\mathrm{i}$ and $\mathrm{j}$, respectively, and $l_{\mathrm{i}}$ and $l_{\mathrm{j}}$ are the number of beads in each site. This expression captures the correct physical behavior as two sites slide laterally by one another, with a maximum occurring at perfect registration. Likewise, as sites move apart along the center of mass displacement vector, the standard exponential decay of the wavefunction overlap is reproduced.

Eq. 2 must be modified for evaluating the charge transfer rates between neighboring sites on the same chain due to the potentially large coupling between bonded conjugated rings. Specifically, the electronic coupling between neighboring backbone beads increases with the alignment in their $\pi$-orbitals and can be expressed in terms of cosine function in the dihedrals between these beads $\left(J_{\text {intra }} \cos \left(\phi_{\mathrm{ij}}\right)\right) .{ }^{34}$ This coupling leads to a modulation of the intramolecular hopping barrier due to orbital mixing, ${ }^{53}$ which is approximated as

$$
\omega_{\mathrm{ij}}=v \exp \left\{-\frac{\left(\Delta E_{\mathrm{ij}}-\lambda_{\mathrm{ij}}\right)^{2}}{4 \pi \lambda_{\mathrm{ij}} k_{B} T}+\frac{\left|J_{\mathrm{intra}} \cos \left(\phi_{\mathrm{ij}}\right)\right|}{k_{B} T}\right\} .
$$

In this expression, the semi-classical Marcus activation energy is recovered when the backbone beads are perpendicular, whereas the barrier can be lowered by up to a factor of $J_{\text {intra }}$ when they are co-planar. The definition of variables $\lambda_{\mathrm{ij}}$ and $\Delta E_{\mathrm{ij}}$ are the same as in Eq. 2, and $v$ is a prefactor associated with the nuclear frequency, ${ }^{54}$ and was set to $10^{15} s^{-1} .53$
A basis of hopping sites localized on individual beads neglects potential delocalization effects and is inappropriate for the evaluation of the Marcus parameters associated with Eqs. 2-4. To account for delocalization, rates calculated using Eq. 5 in an individual bead basis were used to partition contiguous beads into sites comprised of one or more backbone beads. We used the rates, rather than the dihedrals, to demarcate sites because electrostatic factors (e.g., a nearby ion) can also contribute to site localization. Details of site formation are covered in the subsequent section, however this detail affects the manner in which the Marcus parameters, $\lambda_{\mathrm{ij}}$ and $\Delta E_{\mathrm{ij}}$, are evaluated. The reorganization energy, $\lambda_{\mathrm{ij}}$ is the energy associated with the rearrangement of the nuclear coordinates due to charge transfer and consists of internal, $\lambda_{\text {int }}$, and external, $\lambda_{\text {ext }}$, contributions. The internal reorganization energy is associated with the nuclear rearrangement of the sites $i$ and $j$, given by an average of individual internal reorganization energies $\lambda_{i}$ and $\lambda_{j}$, which vary inversely with the size of the site. The values for internal reorganization energy associated with sites of varying number of backbone beads were obtained by fitting a quadratic function to the values for polypyrrole. ${ }^{53}$ The external reorganization energy was approximated using the model introduced by Marcus, ${ }^{55,56}$ obtained by treating the sites as spheres with constant charge density and the solvent as a continuum dielectric,

$$
\lambda_{\mathrm{ext}, \mathrm{ij}}=\frac{e^{2}}{4 \pi \varepsilon_{0}}\left(\frac{1}{\varepsilon_{\mathrm{opt}}}-\frac{1}{\varepsilon_{s}}\right)\left(\frac{1}{2 r_{\mathrm{i}}}+\frac{1}{2 r_{\mathrm{j}}}-\frac{1}{r_{\mathrm{ij}}}\right),
$$

where $r_{i j}, r_{\mathrm{i}}, r_{\mathrm{j}}, \varepsilon_{\mathrm{opt}}$ and $\varepsilon_{s}$ are the distance between the sites, the radii of the sites, the optical frequency dielectric constant, and the static dielectric constant, respectively. $r_{i j}$ is calculated based on the distance between the center of mass of the sites and the radius of a site is taken to be an average of the radius along the backbone and perpendicular to the backbone. For sites that significantly extend along the polymer backbone, the spherical approximation fails, and the expression can produce negative values which were set to zero in this study. Thus, the external reorganization energy decreases with increasing site delocalization until reaching zero. Mixed conducting systems are 
heterogenous, composed of organics and aqueous solvent, and hence, an intermediate dielectric constant of $\varepsilon_{s}=10$ was used. We note that more sophisticated treatments of the external reorganization energy and local dielectric are compatible with the current KMC framework, ${ }^{51}$ however we defer treatment of these to future work.

The site energy difference is given by $\Delta E_{\mathrm{ij}}=$ $E_{j}-E_{i}$, where the individual site energies include contributions from the ionization potential and the local electrostatic environment of each site. The contribution from the ionization potential was modeled as a linear function in inverse length fitted to the values for polypyrrole. ${ }^{53}$ The electrostatic energy contribution to each super-site was modeled on the basis of the CGMD configurations as

$$
E_{\mathrm{elec}, \mathrm{i}}=\sum_{m \subset i} \sum_{n \not \subset i} \frac{q_{m} q_{n}}{4 \pi \varepsilon_{s} \varepsilon_{0} r_{m n}}
$$

where $m$ denotes beads within the site and $n$ denotes beads outside the site. In the charged state, the site has an excess unit charge equally distributed along the polymer (i.e., $1 / l_{i}$ for site length $l_{i}$ ), while the deficit of unit charge is distributed to all other backbone beads in the system. These electrostatic energies were evaluated in LAMMPS by rerunning the CGMD configurations with each site evaluated in the corresponding oxidation state and including long-range interactions and periodic boundary conditions. The charge transfer parameters used in all KMC simulations are summarized in SI.

\subsection{KMC Framework}

Charge transfer is modeled here from the perspective of a single explicit polaron in the mean-field of the other polarons that are present on the polymer. This assumption is reflected in the rate expressions described above, where the electrostatic contributions to the site energies $\left(\Delta E_{\mathrm{ij}}\right)$ are calculated with the residual polymer charge equally distributed to all backbone beads. This approximation neglects polaron correlations and potential coulomb blockade effects that may be relevant at high charge densities, but it is an important reference for establishing how OMIEC morphology and design features affect charge transport. For a single explicit polaron capable of occupying a pre-defined set of polaron sites, the evolution of the charge dynamics is governed by a master equation composed of coupled first-order differential equations

$$
\dot{p}_{\mathrm{i}}=\sum_{\mathrm{j}} k_{\mathrm{ji}} p_{\mathrm{j}}-\sum_{\mathrm{j}} k_{\mathrm{ij}} p_{\mathrm{i}},
$$

where $p_{\mathrm{i}}$ is the probability of the polaron occupying site $\mathrm{i}$ at time $t$, and $k_{\mathrm{ij}}$ is the charge transfer rate between sites as defined in the previous section. Both transient and steady-state solutions to this master equation can be calculated using the kinetic Monte Carlo (KMC) algorithm, which consists of simulating an ensemble of Markov chains with transitions based on Eqs. 8. ${ }^{26}$ This approach is advantageous because it yields both correct steadystate occupation statistics and trajectories that can be analyzed to characterize details of the conduction network. For instance, polaron diffusivities are obtained based on the mean-squared displacement of charges, and graphical analysis can be used to identify resistive bottlenecks in the transport network. Likewise, the KMC framework can be extended include the treatment of more than one explicit polaron.

The definitions of available polaron sites, their site energies, and associated rates are required for the application of KMC. For typical conjugated polymers, polarons are delocalized over multiple backbone units, which makes sites composed of one or more backbone beads in our CG model the relevant basis states for KMC. A typical approach for identifying these delocalized sites from MD simulations is to break each polymer backbone into segments based on deviations in the backbone dihedrals that exceed a threshold. ${ }^{53}$ However, in OMIECs polaron-ion electrostatic interactions also contribute to site localization. To account for this, we use the rates of intramolecular transport, as predicted by Eq. 5 for sites composed of individual backbone beads, as the basis for designating sites. In particular, the intramolecular rates reflect contributions from both dihedral deviations as well as the differences in electrostatics along the backbone. After calculating the charge transfer rates between neighboring beads, the sites are defined based on contiguous groups of backbone beads with a transfer rate above $10^{14} s^{-1}$. These sites are the reference states for Eq. 8 
and charge transfer between sites is modeled using Eq. 2 with size dependent input parameters as described in the previous section.

In a KMC framework, sites and transition rates are held fixed while evaluating charge transport, whereas in the real system, transition rates are a function of time. Rate fluctuations that occur on timescales that are shorter than the characteristic charge transfer rates within the master equation can be incorporated by averaging the rate calculations over a commensurate interval of MD configurations. To characterize relevant shorttimescale fluctuations, we evaluated the autocorrelation functions of the dihedrals $\left(\phi_{i j}\right)$, bonds $\left(r_{i j}\right)$ and electrostatic energies $\left(\Delta E_{\text {elec,ij }}\right)$, shown in Fig. S2 and Fig. S3. We observe that all of these factors decorrelate within 1 ps, and thus use 20 configurations uniformly sampled across a 2 ps interval to average these fluctuations and their contributions to the charge transfer rates.

On timescales longer than the characteristic charge transfer rate, the assumption of a fixed master equation can also break down due to slow diffusion processes or the periodicity of the system (e.g., the polaron traverses many box lengths and the system remains unphysically correlated with the initial state). Webb et al. have recently addressed this issue in the context of polymer electrolytes by incorporating site refresh events into the KMC Markov chains. ${ }^{57}$ In our system, we introduced site refresh events when the charge travelled 10 times the box length, which is between $380-500 \mathrm{~nm}$ in the systems studied here. The site refresh event consisted of (a) rotating the configuration by a random angle about each cartesian axis, in a random order, $(b)$ randomly selecting a polaron site from the configuration as the occupied site, and $(c)$ translating the occupied site to the position of the originally occupied site. A refresh criteria for addressing polymer diffusion was not used, since we observed that all of our simulations of dense polymer networks reached the Fickian regime (for charge transport) well within the diffusion timescale of the polymers and ions. It is possible that for less viscous systems, a site refresh related to the fastest diffusion timescale would be required.

For each of the hydration and oxidation conditions that were studied, a total of 60 uncorre- lated configurations were characterized by KMC. Specifically, 20 configurations were sampled every five ns from three independent MD simulations. These configuration counts exclude the additional 0.1 ps spaced configurations used for averaging charge transfer rates. For each configuration, 20 KMC charge transport trajectories were simulated for $150 \mathrm{~ns}$. The mean square displacement (MSD) of the charge was calculated for each configuration and averaged over all 60 configurations. The selfdiffusion coefficient was obtained from the MSD using the relation,

$$
D=\lim _{t \rightarrow \infty} \frac{1}{6} \frac{d}{d t}\left\langle|\mathbf{r}(t)-\mathbf{r}(0)|^{2}\right\rangle
$$

where $\mathbf{r}(t)$ is the position vector of the center of mass of the site occupied by the charge at time $t$. The angular brackets in Eq. 9 indicate an average over all polarons and time origins. Numerically, Eq. 9 was evaluated by identifying the Fickian regime of the MSD curves (i.e., where the curves exhibited a consistent $\log (\mathrm{MSD})$ vs $\log (t)$ of one), then performing a least-squares linear regression to the interval from 10ns-100ns. The mobility was obtained from the diffusivity using the Einstein relation,

$$
\mu=\frac{e D}{k_{B} T},
$$

where $e$ is the unit electron charge.

\section{Results and Discussion}

\subsection{Effect of Hydration Level}

To establish the structural changes that accompany hydration of our model OMIEC, we characterized the joint-probability distribution for $\pi-\pi$ stacking and radial separation between the polymer backbones as a function of hydration level while fixing the oxidation level at the intermediate value of $+0.2 \mathrm{e}$ per backbone bead (Fig. 4). In these distributions, the $\mathrm{x}$ and $\mathrm{y}$ axes represent the distance between backbone beads on different chains $\left(r_{\mathrm{ij}}\right)$ and their $\pi-\pi$ alignment $\left(s_{\mathrm{ij}}=\mathbf{v}_{\mathrm{i}} \cdot \mathbf{v}_{\mathrm{j}}\right)$, respectively, while the color represents their joint probability 

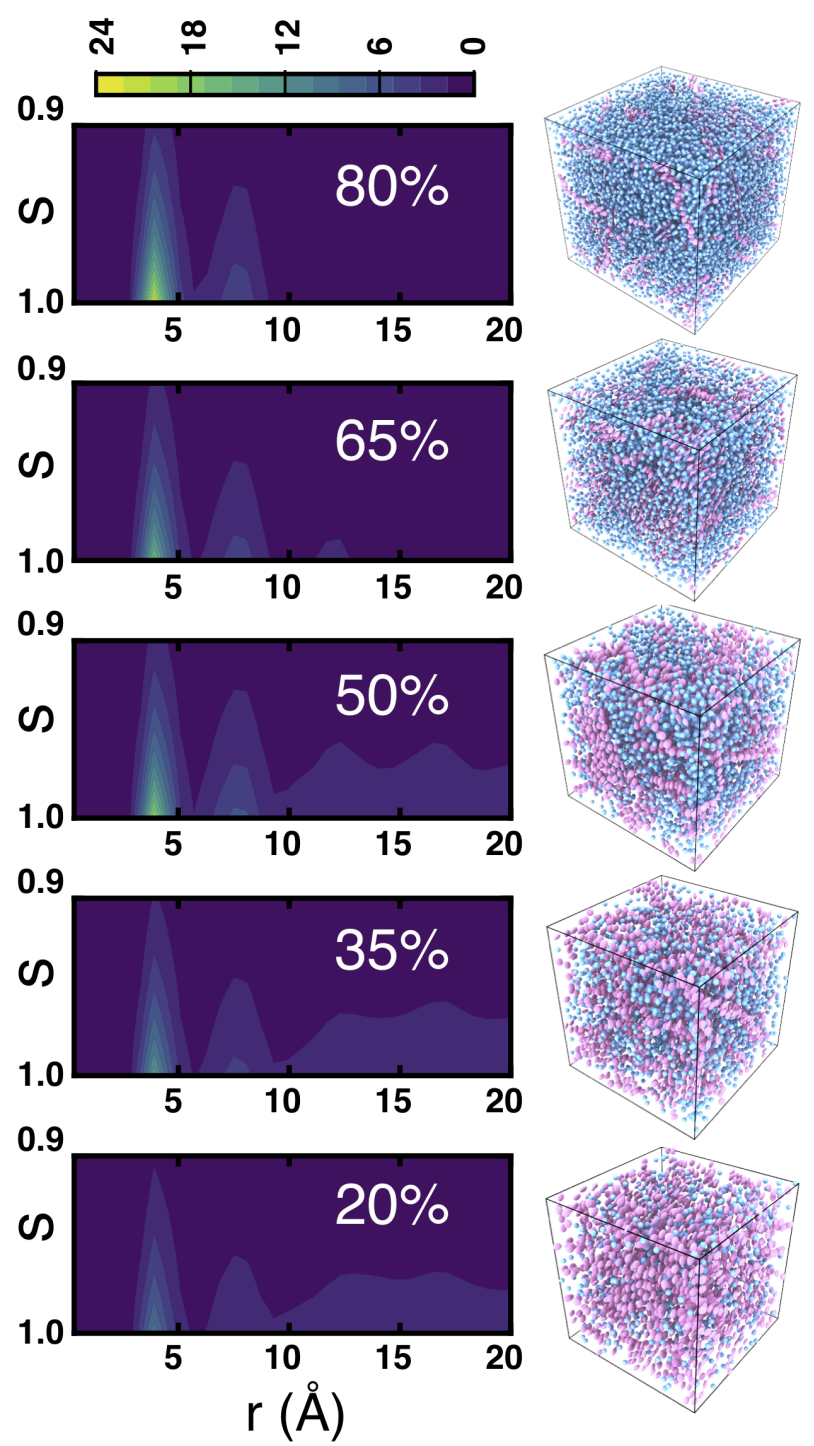

Figure 4: $\pi-\pi$ stacking probability presented as a joint probability distribution of distance between the backbone beads on the $\mathrm{x}$-axis and $\pi-\pi$ alignment on the $y$-axis. Trajectory snapshots are shown to the right, where the pink and blue beads are backbone beads and water, respectively. Other components are omitted for clarity.

distribution given by

$$
g(r, s)=\frac{V}{4 \pi r^{2} \mathrm{~N}_{\mathrm{p}}} \sum_{\mathrm{i}=1}^{N} \sum_{\mathrm{j}=1}^{N} \delta\left(r-r_{\mathrm{ij}}\right) \delta\left(s-s_{\mathrm{ij}}\right),
$$

where $N_{p}$ is the total number of pairs of backbone beads in the system, excluding pairs within the same molecule. These distributions are averaged over configurations parsed from multiple independent trajectories after equilibration of the density, potential energy, and translational diffusion of the chains, and thus reflect an effort to sample authentic equilibrium configurations within the limits of the MD simulations.

Prominent $\pi-\pi$ stacking is apparent at all hydration levels (i.e., $s_{\mathrm{ij}}>0.9$ in all of the peaks), which is consistent with conjugated polymers in general and the backbone anisotropy of our model OMIEC. All of the systems also exhibit peaks at multiples of $\sim 4 \AA$, which is indicative of aggregates of $\pi-\pi$ stacked polymers. In contrast, some lamellar organization occurs at low hydration levels $(\sim 10 \AA)$ but overall it is weak in comparison with the nearby higher-order $\pi-\pi$ stacking peak at this oxidation level. We also observe an abrupt transition between the $50 \%$ and $65 \%$ hydration levels that corresponds to the dissolution of the polymer aggregates. Specifically, at hydration levels of $65-80 \%$, we do not find any features at distances greater than $1.5 \mathrm{~nm}$, as the polymers are separated by large clusters of water, while at hydration levels $\leq 50 \%$ dense percolating polymer networks are formed. We also observe that the $\pi-\pi$ alignment increases with increasing hydration level. ${ }^{27}$ This reflects the configurational freedom of the smaller aggregates to reorganize into highly aligned structures, whereas packing defects frustrate chain registration at low hydration levels. Interestingly, at the intermediate hydration level of $50 \%$, both percolation and relatively strong $\pi-\pi$ alignment are achieved. This structural analysis suggests a potential trade-off between chain alignment and the number of conductive pathways as the hydration level is reduced, wherein highly hydrated morphologies (e.g., $\rho_{\mathrm{H}_{2} \mathrm{O}}=50 \%$ ) exhibit relatively few pathways that are highly aligned while less hydrated morphologies exhibit a larger number of conductive pathways that are on average more disordered.

To clarify the distinct spatial distributions of the electrolyte phase within each system, we also analyzed the size and distribution of water clusters (Fig. 5). Clusters were defined using nearestneighbor hierarchical clustering with a threshold of $5.8 \AA$, which is $10 \%$ above the Van der Waals equilibrium distance for the Martini water model (i.e., all water molecules in a cluster are within the threshold of at least one other member of the cluster, and molecules in distinct clusters are separated by at least the threshold distance). The ob- 


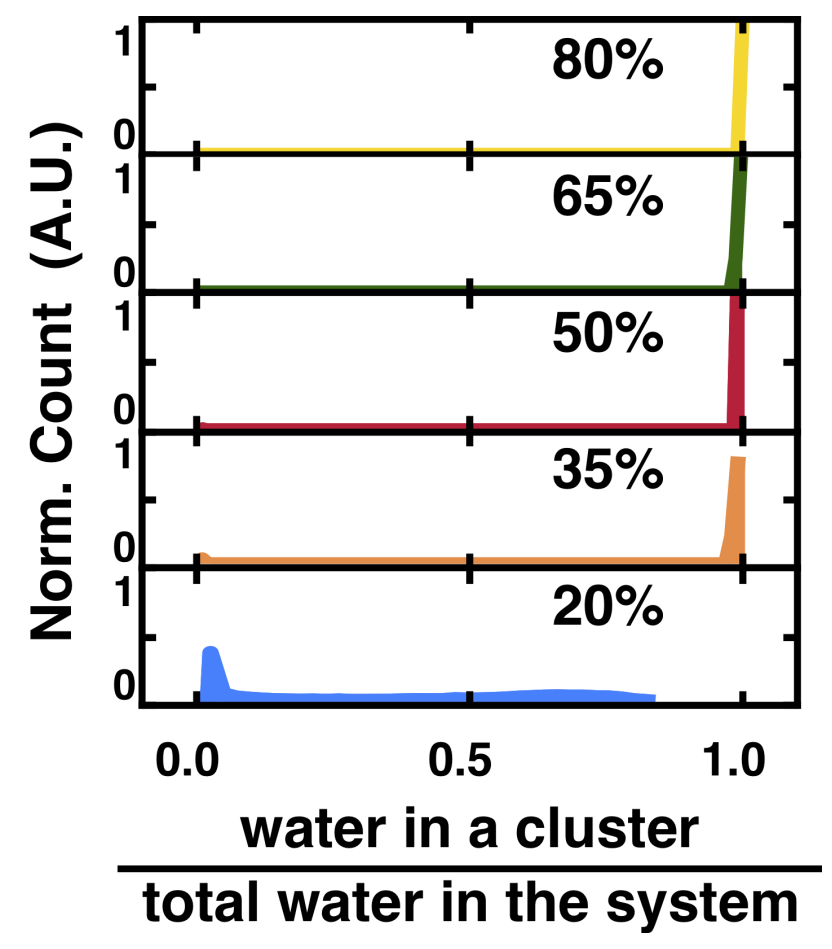

Figure 5: Distribution of water clusters for different hydration levels. The number of molecules in a cluster normalized the by total number of molecules is plotted on the x-axis and the number of its occurrences normalized by the maximum number of possible occurrences is plotted on the $y$ axis.

served cluster distributions are bimodal, consisting of either small water clusters with very few molecules, or a single large percolating cluster. Interestingly, no moderately sized clusters are observed, even at low hydration levels. Rather, for all hydration levels $\geq 35 \%$, the electrolyte phase percolates throughout the volume of the polymer. This indicates that the electrolyte spontaneously percolates throughout the polymer phase through channel formation, rather than through the coalescence of gradually increasing water clusters. In contrast, a recent computational study of PEDOT: $\operatorname{Tos}^{27}$ reports a gradual increase of water cluster size through coalescence which can be attributed to the tosylate dopants dispersed throughout the system.

To determine the impact of these distinct morphologies on charge transport, KMC simulations were performed on each system (Fig. 6). For the $65 \%$ and $80 \%$ hydration level simulations, subdiffusive behavior is observed, which is consis-

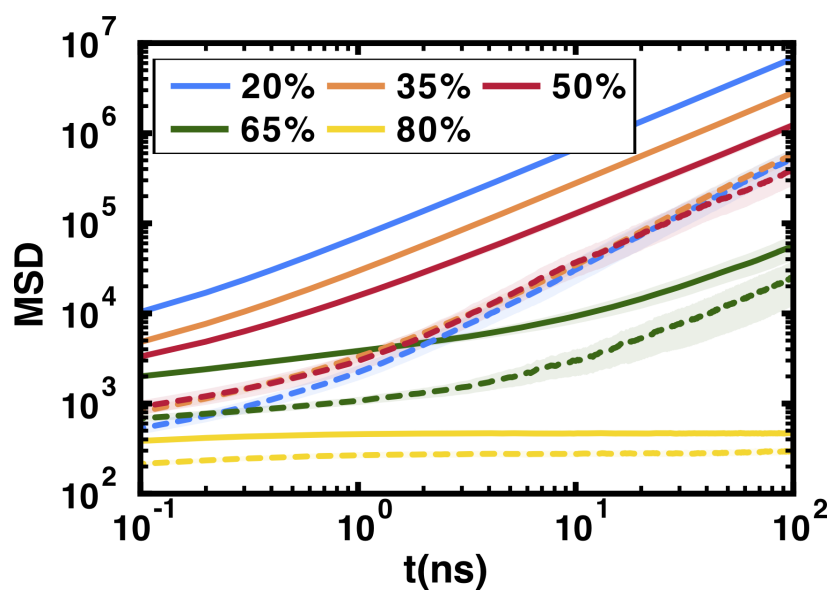

Figure 6: Mean square displacement of charge with intermolecular rates excluding (solid lines) and including (dashed lines) electrostatic energy component at different hydration levels. Standard deviations calculated across the sampled configurations are shown in the shaded regions.

tent with the dissolution of the polymers and lack of macroscopic percolation. These cases are excluded from further discussion of charge transport, however we highlight the recent description of scaling laws in this concentration regime by Sing et al. ${ }^{58}$ Additionally, a comparison was performed of KMC results with and without electrostatic contributions to the site energy differences to elucidate the impact of ion-polaron coupling on the charge transport. In all of the simulations, the polarons are delocalized along the entire polymer chain based on the intramolecular rates (as described in Section II.F) and each polymer served as a single site for intermolecular charge transport. Comparing the polaron mean-squared displacement (MSD) in the two cases (Fig. 6), illustrates that including ion-polaron electrostatics suppresses charge transport at all hydration levels. This is intuitive, as all else being equal, electrostatics introduce energetic disorder that reduces charge transport rates. However, the results also reveal that the ion-polaron electrostatics suppress charge transport more strongly at low hydration levels, with the consequence that all of the dense systems exhibit comparable polaron mobilities when electrostatics are included. Comparing the quantitative trends within each series of simulations reveals several distinct factors that contribute to the observable polaron mobility. 
When ion-polaron electrostatics are excluded from the simulations, the polaron diffusivity monotonically decreases with increasing hydration level. Based on the previous structural analysis, decreasing hydration leads to relatively decreased $\pi-\pi$ alignment, however this is countervailed by an increase in the number of conductive pathways as the polymer network densifies. These two factors are reflected in the distributions of charge transfer rates (Fig. 7a) and site connectivity (Fig. 7b) within each system. The impact of $\pi-\pi$ alignment is reflected in a peak at high rates within the rate distributions of the $35 \%$ and $50 \%$ systems, whereas the impact of density is reflected in an increase in the site connectivity of the $20 \%$ system. Based on the observed trends in polaron mobility (Fig. 6), site connectivity outweighs the impact of $\pi-\pi$ alignment with respect to increasing polaron mobility. As the connectivity increases, the distinct sites visited both at short (Fig. 7c) and long (Fig. 7d) timescales increase, representing relatively reduced trapping and percolative transport in the highest mobility $20 \%$ system. In contrast, at the $50 \%$ hydration level, many trajectories involve only a small fraction of sites which is indicative of poor connectivity within the transport network despite high charge transfer rates within localized subnetworks of the morphology.

The forgoing examination of charge transport when polaron-ion electrostatics are excluded illustrates the complex interplay between morphology and site connectivity that underpins the ostensibly straightforward trend that increasing hydration reduces polaron mobility. When ion-polaron electrostatics are included in the KMC simulations, these factors are also present, but they compete with the site energy disorder associated with the spatial distribution of the electrolyte. Comparing the rate distributions with and without ionpolaron electrostatics, reveals that electrostatic heterogeneity suppresses the charge transport rates in all systems and the peaks at high rates observed for the $35 \%$ and $50 \%$ hydration levels are greatly reduced (Fig. 7a). Including ion-polaron electrostatics also results in a dramatic reduction in the site connectivity of the $20 \%$ hydrated system, such that all of the dense polymer systems exhibit comparable connectivities (Fig. 7b). This reduction in overall connectivity also leads to a reduction in the (a)

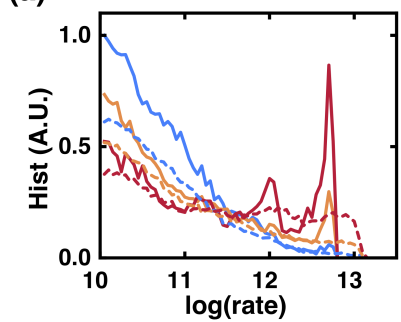

(b)
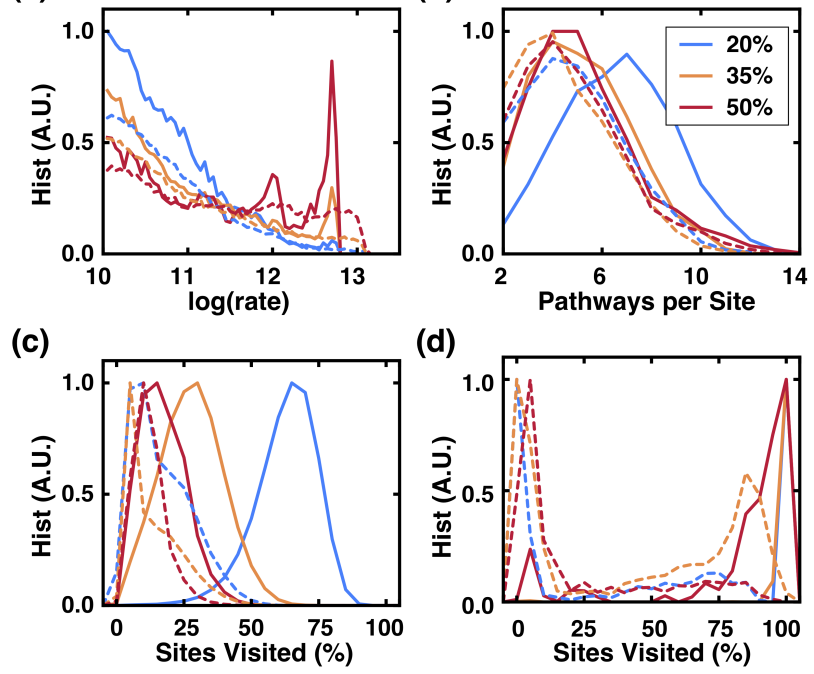

(d)

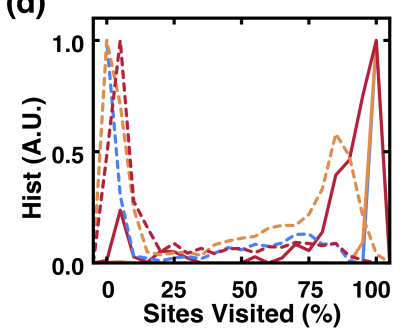

Figure 7: Distribution of (a) intermolecular rates and (b) pathways per site, (c) distinct sites visited over 500 jumps and (d) distinct sites visited over last 75ns trajectory, when excluding (solid lines) and including (dashed lines) electrostatic energy component at different hydration levels. The distributions (a-b) are normalized by the maximum value across all plots and (c) is normalized by the maximum value within each plot.

fraction of sites that participate in charge transport relative to when ion-polaron electrostatics are excluded (Fig. 7c-d). The contraction in the number of sites visited reflects trapping of the polaron to clusters of sites with similar electrostatic energies (Fig. S6). This is further evidenced by the occurrence of sub-diffusive behavior in all of the dense networks when ion-polaron electrostatics are included in the simulations (Fig. 6). The consistent picture that emerges is that ion percolation within the polymer phase results in localization of charge transport to a subnetwork of sites with similar electrostatic environments. Remarkably, these subnetworks are similar in all of the dense systems with the net result that the polaron mobility is relatively constant with respect to hydration level. This effect has also been observed experimentally in some OMIECs, where the measured hole mobility is insensitive to swelling caused by varying the anion in the electrolyte ${ }^{59}$ and varying the electrolyte concentration. ${ }^{60}$

To directly interrogate the changes in electrostatics that accompany ion percolation within the polymer, we also analyzed the distribution of elec- 
trostatic energies of the polaron sites and the ionpolymer backbone RDF at each hydration level (Fig. 8). Comparing the RDFs, all systems exhibit a prominent nearest-neighbor peak and periodic features with a spacing of $\sim 4.5 \AA$, which is consistent with ion intercalation within the polymer aggregates. At high hydration levels (i.e., $\rho_{\mathrm{H}_{2} \mathrm{O}} \geq 65 \%$ ) a small decrease in the first RDF peak is accompanied by an increase in the later peaks, which is due to a weak preference for ions to remain external to the more highly ordered polymer aggregates after dissolution (Fig. 8 (c-e)). Although all systems display a similar degree of ion mixing based on the RDFs, ${ }^{61}$ the $20 \%$ system exhibits a much broader distribution of electrostatic site energies compared with the $35 \%$ and $50 \%$ hydrated systems (Fig. 8b). This can be rationalized by the earlier observation that the electrolyte phase fails to fully percolate in the $20 \%$ system, which leads to electrostatic heterogeneity and limited electrostatic screening. The resulting electrostatic induced site energy disorder suppresses charge transfer rates and is consonant with the reduced site connectivity of the $20 \%$ system when ion-polaron electrostatics are included.

In most mixed conducting applications, the mobility of ions is critical to mediate reversible doping of the semiconducting polymer. Moreover, ion diffusion is typically much slower than electron diffusion ${ }^{62,63}$ and is the rate-limiting step in (de)doping. The ionic mobility was thus characterized in each system to determine the complementary impact on ion dynamics as the hydration level is varied (Table 1). It is established that Martini dynamics are accelerated by approximately a factor of four in comparison with all-atom simulations. ${ }^{35,64}$ Thus, the reported ionic mobilities have been scaled by a factor of 0.25 relative to the raw MD trajectories. The polaron mobility is between one and two orders of magnitude larger than the ionic mobility for the dense polymer systems (i.e., $\rho_{\mathrm{H}_{2} \mathrm{O}} \leq 50 \%$ ), however at higher hydration levels the electronic mobility is limited by the dissolution of polymer aggregates and the loss of macroscopic percolation pathways. In contrast, the ionic mobility monotonically increases with respect to hydration level, ${ }^{65}$ albeit with distinct behaviors above and below the electrolyte percolation threshold. Specifically, for $\rho_{\mathrm{H}_{2} \mathrm{O}} \geq 35 \%$, the ion diffusivity increases approximately linearly with respect to hydration level, while the $20 \%$ system exhibits an abrupt three-fold reduction in ion mobility. This is consistent with the loss of electrolyte percolation in the $20 \%$ system, resulting in macroscopic ion diffusion being restricted by reorganization of the polymer phase. In comparison, a recent computational study of PEDOT:TOS ${ }^{27}$ reports an exponential increase in ion diffusivity with hydration level, while a recent experimental study on OMIECs with varying side-chains report a sub-exponential trend. ${ }^{63}$ These results highlight the importance of establishing co-percolating electronic and ionic phases within the mixed conductor, and suggest that polymer design strategies focusing on electrolyte management may be critical to striking the optimal balance. We also note that since comparable electronic mobilities were observed in all of the dense systems, we do not observe a significant trade-off between ionic mobility and electronic mobility in this polymer. Specifically, by maintaining hydration levels between $35-50 \%$, through either side-chain or morphology engineering, optimal electronic mobility and ionic mobility within an order of magnitude of the bulk electrolyte can be achieved.
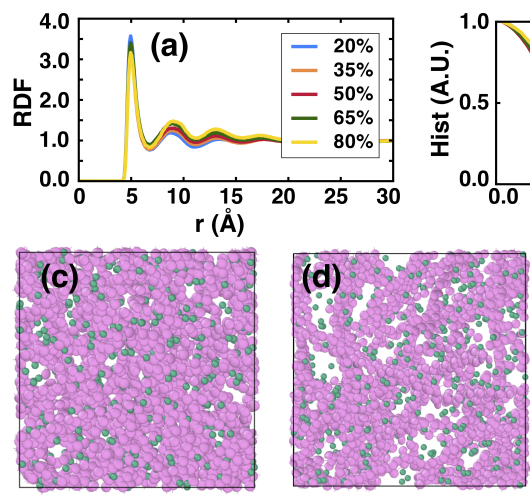
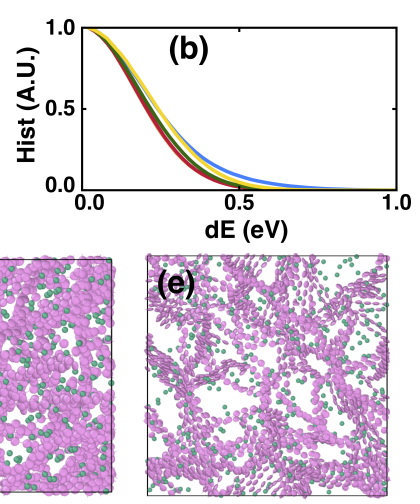

Figure 8: (a) Histogram of the electrostatic site energy differences and (b) ion-backbone RDF for all energy differences; snapshot of the system showing ion (green) distribution around polymer (pink) for hydration level (c) $20 \%$, (d) $50 \%$ and (e) 80 $\%$. The distributions are normalized by the maximum value across all plots. 
Table 1: Summary of transport coefficients at different hydration levels with a fixed oxidation level of 0.2 per backbone unit.

\begin{tabular}{|l|l|l|l|}
\hline$\rho_{\mathrm{H}_{2} \mathrm{O}}^{a}$ & $\mu_{+}^{b}$ & $\mu_{+, E S}^{c}$ & $\mu_{-}^{d}$ \\
\hline $20 \%$ & 14.30 & 1.03 & 0.0081 \\
$35 \%$ & 6.08 & 1.33 & 0.0224 \\
$50 \%$ & 2.94 & 0.87 & 0.041 \\
$65 \%$ & 0.13 & 0.07 & 0.0696 \\
$80 \%$ & - & - & 0.1004 \\
\hline \multicolumn{4}{|c|}{ The hydration level in weight percent. } \\
${ }^{b}$ Polaron mobility with excluded electrostatics, \\
\multicolumn{4}{c}{$10^{-3} \mathrm{~cm}^{2} \mathrm{~V}^{-1} \mathrm{~s}^{-1}$. } \\
\multicolumn{4}{c}{$10^{-3} \mathrm{~cm}^{2} \mathrm{~V}^{-1} \mathrm{~s}^{-1} \cdot$} \\
\end{tabular}

\subsection{Effect of Oxidation Level}

In this section, we inspect the changes in the structural and transport behaviors of the model OMIEC upon changing the oxidation level of the polymer, while keeping the hydration level fixed at $50 \%$. To help orient the reader, the same analyses are performed as in the previous section and discussed in the same order.

To characterize the impact of oxidation level on morphology, the joint probability distribution for $\pi-\pi$ stacking and radial separation between the polymer backbones were analyzed (Fig. 9). No features are observed at very high oxidation levels $(\geq+0.5 e)$ as the polymer completely dissolves due to repulsive inter-chain electrostatics. At lower oxidation levels $(\leq+0.2 e)$, the polymers assemble into well stacked $\pi-\pi$ aggregates with a prominent nearest-neighbor peak and additional peaks at multiples of $\sim 4 \AA$. Although all of the dense systems are well-ordered, a small but persistent increase in $\pi-\pi$ alignment is apparent in the first two peaks as the oxidation level decreases. Additionally, for oxidation levels $\leq$ $+0.2 e$, a prominent lamellar stacking peak occurs at $\sim 10 \AA$ and larger aggregates are formed, ${ }^{27}$ as indicated by the higher probabilities at large radial separations. These distinct changes in morphology indicate that a combination of repulsive inter-chain electrostatics and electrolyte percolation affect the inter-chain disorder and aggregate dimensions as the oxidation level increases. This interplay is also qualitatively apparent in the swelling behavior of many OMIECs upon doping. ${ }^{17,20,66}$
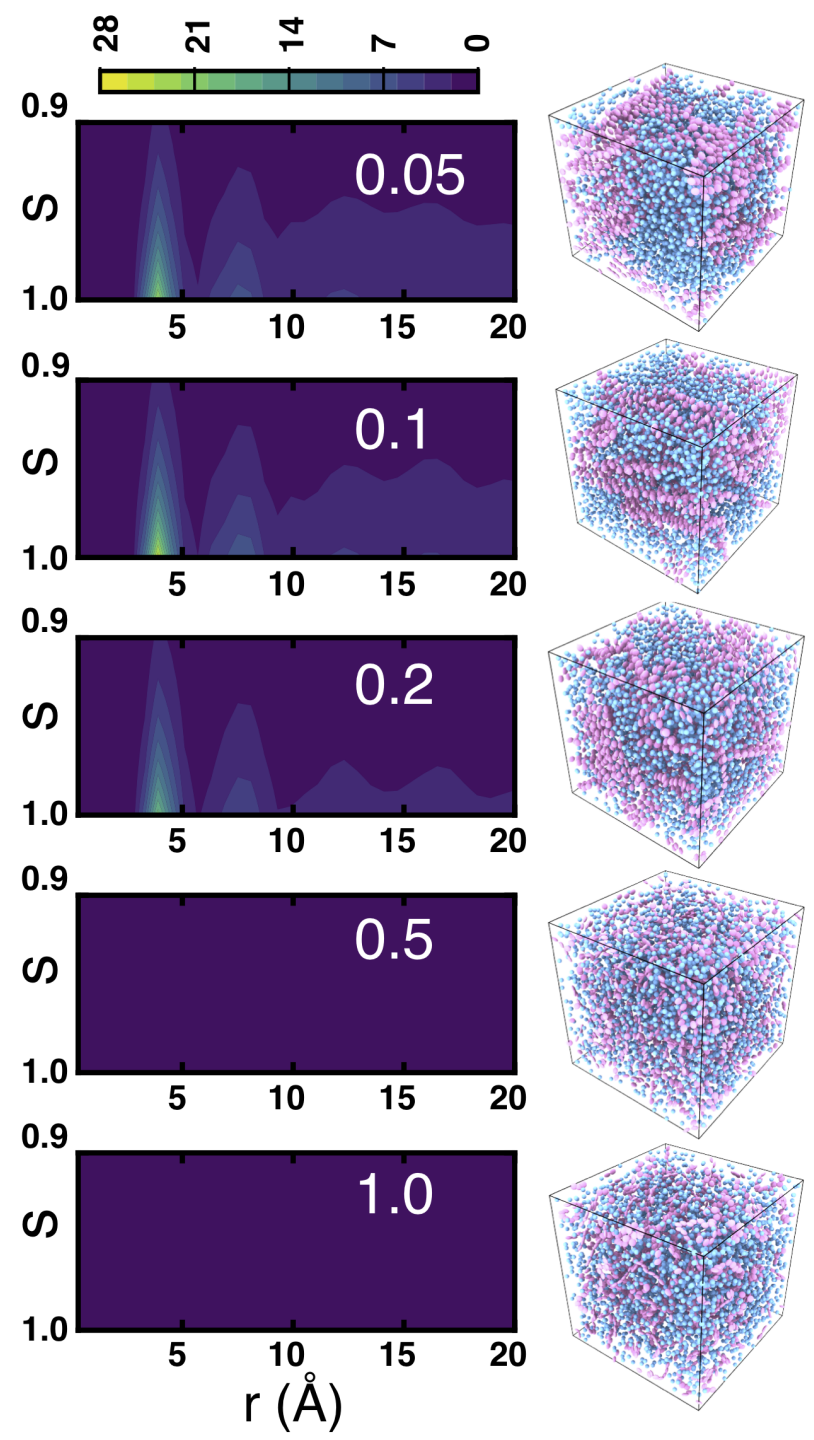

Figure 9: $\pi-\pi$ stacking probability presented as a joint probability distribution of distance between the backbone beads on the x-axis and $\pi-\pi$ alignment on the y-axis. Trajectory snapshots are shown to the right, where the pink and blue beads are backbone beads and water, respectively. Other components are omitted for clarity.

The change in OMIEC morphology with respect to oxidation is also reflected in the distribution of the size of water clusters (Fig. 10). At hydration levels of $50 \%$, all of the systems exhibit a large percolating electrolyte phase; however, the probability of forming a single large percolating cluster decreases as the oxidation level decreases. This reflects the limited percolation of electrolyte through the weakly charged aggregates, which results in a small population of disconnected water 
clusters. Thus, the solvation and morphology of the polymer system, although generally associated with the side-chain chemistry and processing, also exhibits a complex dependence on the extent of polymer doping.

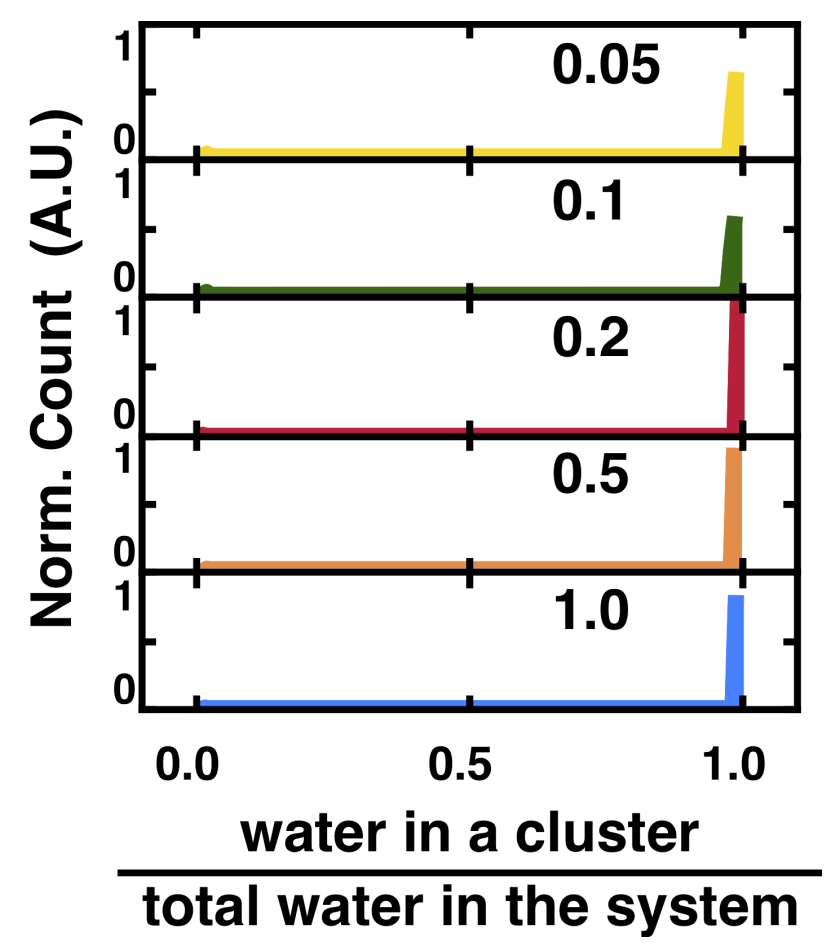

Figure 10: Distribution of water clusters for different oxidation levels. The number of molecules in a cluster normalized the by total number of molecules is plotted on the x-axis and the number of its occurrences normalized by the maximum number of possible occurrences is plotted on the $y$ axis.

To establish how these morphological differences impact polaron transport, KMC simulations were used to characterize the polaron MSD at each oxidation level (Fig. 11). To decouple the role of electrostatics and morphology, a comparison of the polaron MSD with and without ion-polymer electrostatics is also presented. In all cases, we observe that the polaron is delocalized along the entire backbone, and thus polaron transport occurs via hopping between distinct chains. For oxidation levels $q \geq+0.5 e$, the polymers are completely dissolved and are excluded from further discussion. Similar to the simulations that were performed with respect to varying hydration level, the inclusion of ion-polymer electrostatics suppresses polaron transport in all cases. However, unlike the

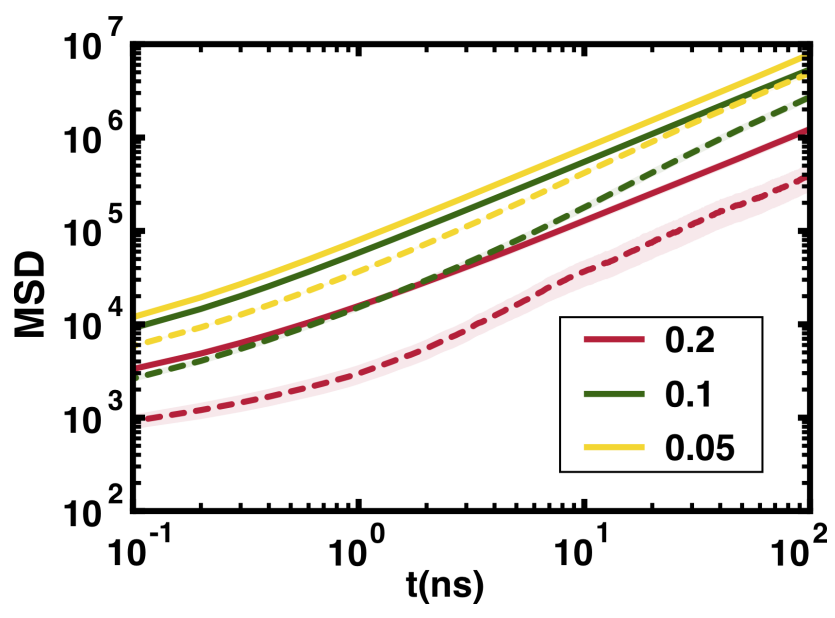

Figure 11: Mean square displacement of charge with intermolecular rates excluding (solid lines) and including (dashed lines) electrostatic energy component at different oxidation levels. Standard errors calculated across the sampled configurations are shown in the shaded regions.

case of varying hydration level, a monotonic decrease in polaron mobility with respect to oxidation level is observed both with and without electrostatics. A comparison of the quantitative details reveals several distinct mechanisms by which oxidation level modulates the polaron mobility in comparison with hydration level.

When ion-polaron electrostatics are excluded from the simulations, the polaron diffusivity monotonically decreases with increasing oxidation level. Based on the previous structural analysis, decreasing oxidation leads to relatively increased $\pi-\pi$ alignment and the formation of larger lamellar aggregates. These two factors are reflected in the distributions of charge transfer rates (Fig. 12a) and site connectivity (Fig. 12b) within each system. All of the dense systems exhibit a peak at high rates, with an increase in site connectivity as oxidation is reduced. We note that despite the relative decrease in $\pi-\pi$ alignment of individual backbone beads with oxidation level, the $+0.2 \mathrm{e}$ system exhibits the largest unnormalized number of high rate pathways, which suggests that site alignment-versus bead alignment-is in fact marginally improved at higher oxidation levels. Thus, similar to the effect of hydration, charge transfer rates are reduced but site connectivity is increased with reduced oxidation, which leads to the observed increase in polaron mobility. This 
trend is also consistent with the observed increase in sites visited at both short and long timescales (Fig. 12c-d) as the oxidation level is reduced. (a)

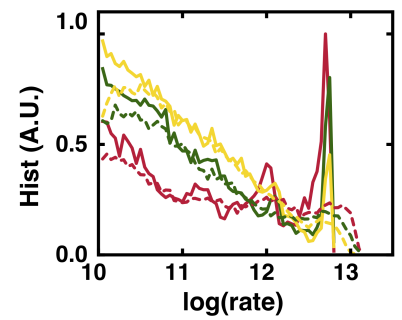

(c)

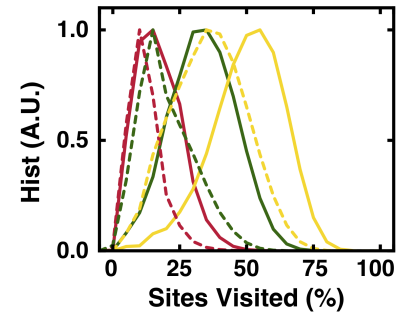

(b)

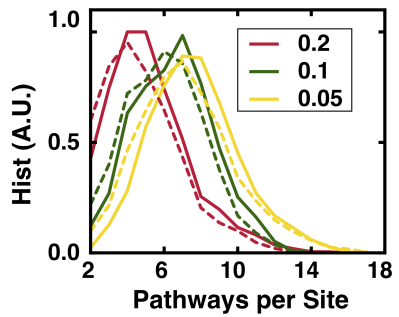

(d)

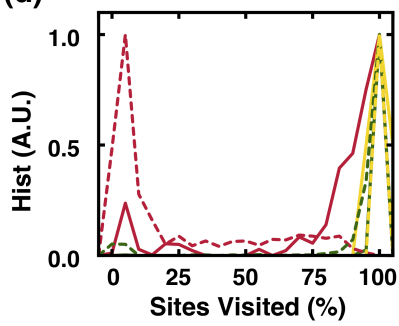

Figure 12: Distribution of (a) intermolecular rates and (b) pathways per site, (c) distinct sites visited over 500 jumps and (d) distinct sites visited over last $75 \mathrm{~ns}$ trajectory, when excluding (solid lines) and including (dashed lines) electrostatic energy component at different oxidation levels. The distributions (a-b) are normalized by the maximum value across all plots and (c) is normalized by the maximum value within each plot.

When ion-polymer electrostatics are included in the KMC simulations, the polaron transport is suppressed in all cases but the behavior of the $q=+0.2 e$ system is qualitatively altered. Due to the additional electrostatic site disorder, all of the systems apparently exhibit a similar suppression of fast charge transport rates (Fig. 12a) and a minor decrease in the mean site connectivity ( Fig. 12b). However, comparing the site visitation histograms, the $q=+0.2 e$ system exhibits dramatically increased polaron trapping compared with the less oxidized systems ( Fig. 12c-d). We interpret this result to indicate that although the mean site connectivity is only marginally reduced in the $q=+0.2 e$ system, the pathways that are suppressed by the inclusion of electrostatics are inter-aggregate connections. In contrast, the lower oxidation level systems exhibit similar percolative polaron transport both with and without the inclusion of ion-polymer electrostatics. It should be noted that this disparity in transport would be mitigated at lower hydration levels. Thus, the polaron transport is determined by an interplay of oxidation level and hydration level, which are experimentally coupled by electrolyte gating, polymer chemistry, and processing conditions.
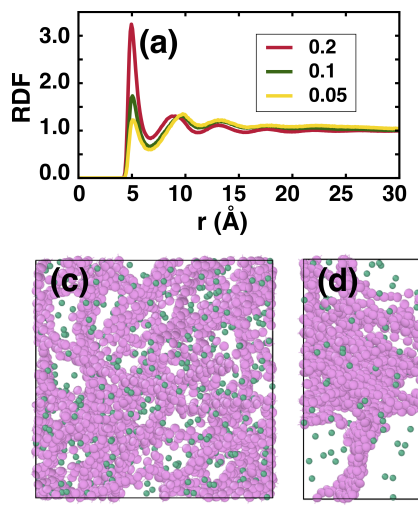

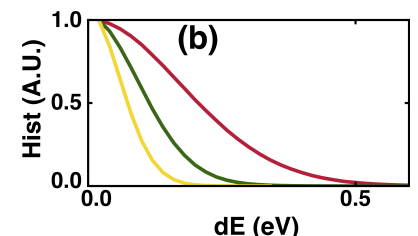

$\mathrm{dE}(\mathrm{eV})$

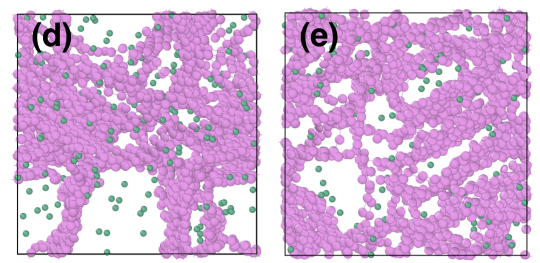

Figure 13: (a) Histogram of the electrostatic site energy differences and (b) ion-backbone RDF for all energy differences; snapshot of the system showing ion (green) distribution around polymer (pink) for oxidation level (c) 0.2 , (d) 0.1 and (e) 0.05 . The distributions are normalized by the maximum value across all plots.

Unlike the hydration level series, we find significant differences in ion percolation in the polymer phase as oxidation level changes. Comparing the ion-polymer RDFs, ion intercalation within the polymer aggregates decreases with decreasing oxidation level (Fig. 13a), which is consistent with the earlier structural and water cluster analyses. Note that the lower oxidation levels have fewer total ions in the simulation box; nevertheless, a smaller fraction of ions intercalate within the polymer phase (Fig. 13c-e). The distinct ion distributions are also evident in the much higher electrostatic heterogeneity observed in the $q=+0.2 e$ system (Fig. 13b). Due to a combination of increased ion density and the fact that the ions intercalate rather than surround the polymer aggregates, high oxidation levels induce electrostatic disorder that strongly suppresses the polaron mobility (Table 2 ). We note that the electronic conductivity rather than the mobility is often relevant for applications. For the model OMIEC investigated here, the polaron mobility is reduced approximately eight-fold in the the $q=+0.2 e$ system compared with the $q=+0.1 e$ system, suggesting that maximum con- 
ductivities would actually be obtained by operating at lower oxidation levels (i.e., $q=0.05-0.1 e$ ).

Table 2: Summary of transport coefficients at different oxidation levels with a fixed hydration level of $50 \%$.

\begin{tabular}{|l|l|l|l|}
\hline$q_{B}^{a}$ & $\mu_{+}^{b}$ & $\mu_{+, E S}^{c}$ & $\mu_{-}^{d}$ \\
\hline 1.0 & - & - & 0.0013 \\
0.5 & - & - & 0.032 \\
0.2 & 2.94 & 0.87 & 0.041 \\
0.1 & 11.82 & 6.53 & 0.0536 \\
0.05 & 16.31 & 10.09 & 0.0551 \\
\hline \multicolumn{4}{|c|}{ The oxidation level per backbone unit. } \\
Polaron mobility with excluded electrostatics, \\
10 $10^{-3} \mathrm{~cm}^{2} \mathrm{~V}^{-1} \mathrm{~s}^{-1}$. \\
Polaron mobility with included electrostatics, \\
10 $0^{-3} \mathrm{~cm}^{2} \mathrm{~V}^{-1} \mathrm{~s}^{-1} \cdot$ \\
Anion mobility, $10^{-3} \mathrm{~cm}^{2} \mathrm{~V}^{-1} \mathrm{~s}^{-1}$.
\end{tabular}

Finally, the ionic mobility was characterized in each system to determine the effect on ion dynamics as the oxidation level is varied (Table 2). As the oxidation level increases, the ion mobility monotonically decreases. At the highest oxidation level $(q=+1.0 e)$, strong ion-polymer coupling leads to an acute non-linear suppression of the ion mobility. However, at the lower oxidation levels that are more representative of experimentally attainable doping, the ion mobility is only weakly sensitive to the oxidation level and also substantially lower than the polaron mobility. This weak sensitivity is notable in light of the distinct morphologies and electrolyte percolation characteristics as oxidation is varied. This reflects the fact that at the $50 \%$ hydration level, the electrolyte still exhibits macroscopic percolation in all of the investigated systems. At lower hydration levels it is possible that the oxidation level has a stronger impact on ion mobility.

\section{Conclusions}

Ionic and electronic transport are coupled within OMIECs through a complex interplay of morphological and electrostatic changes that accompany doping. Although the molecular details of these features are consequential for rational OMIEC design, they occur on length and timescales that are difficult to experimentally probe. In this contribution, we have presented a modeling framework that addresses this characterization gap while retaining sufficient chemical flexibility and molecular physics to resolve structure-function relationships. Specifically, polymer and electrolyte dynamics are modeled at the coarse-grained level using the Martini force-field supplemented by an anisotropic Gay-Berne description of the conjugated OMIEC backbone, while polaron dynamics are modeled as hopping between localized sites with rates and site energies determined by sampling configurations from the CG trajectories. In combination, these approaches are capable of simultaneously probing the molecular details of morphology and macroscopic conduction as a function of polymer identity, electrolyte concentration, and doping level.

As a demonstration of this framework, we have presented a comprehensive characterization of ion and polaron transport in a model OMIEC as a function of oxidation and hydration levels. In each case, qualitative differences in polaron dynamics occurred when ion-polaron electrostatics were excluded from the model, which reinforces the critical role of ion-polaron coupling on local transport behaviors. The simulations also recapitulate several intuitive trends, including the suppression of polaron transport by electrolyte induced morphological disorder and the increase in ion mobility with hydration level, while revealing several mechanisms that can mitigate trade-off between ion transport and polaron transport. For instance, swelling by the electrolyte is accompanied by increased screening and a decrease in deep electrostatic traps that results in comparable polaron mobilities at low to moderate hydration levels. Additionally, we observed a non-linear suppression of polaron mobility as a function of oxidation level that indicates an optimum doping level for this system. This latter result occurs despite the fact that the polaron simulations are performed in a meanfield approximation of other polarons, and thus exclude polaron-polaron correlations and Coulomb blockade effects that are likely to further suppress transport at high oxidation levels.

With respect to the design of OMIECs, the current work elucidates several molecular scale factors that are potentially subject to rational design. 
The results highlight near-term opportunities for tuning side-chain hydrophilicity, density, and sequence to achieve electrolyte percolation without suppressing inter-aggregate polaron transport, and possibly to tune equilibrium polymer-ion configurations. On the longer term, engineering polymer aggregate dimensions and connectivity are potentially achievable by exploring electrolyte additives or tuning polymer-polymer interactions. The presented computational framework is capable of decoupling morphological features of the system such as $\pi-\pi$ overlap and connectivity of the polymer network, and further account for their relative contribution to charge transport in the system. Although the current work is limited to results for just one model OMIEC that serves primarily as a validation activity for the modeling framework, these relationships are being explored in ongoing work to establish the general structure-function relationships of OMIECs.

\section{Acknowledgments}

This work used the Extreme Science and Engineering Discovery Environment (XSEDE), which is supported by National Science Foundation grant number ACI-1548562. Simulations were performed on the Comet supercomputer at the University of California, San Diego, under the allocation TG-CHE190014. Acknowledgment is made to the Donors of the American Chemical Society Petroleum Research Fund for partial support of this research. B. M. S. acknowledges support from the Dreyfus Program for Machine Learning in the Chemical Sciences and Engineering during this project.

\section{References}

(1) Paulsen, B. D.; Tybrandt, K.; Stavrinidou, E.; Rivnay, J. Organic mixed ionic-electronic conductors. Nature Materials 2019, 1-14.

(2) Zhao, F.; Shi, Y.; Pan, L.; Yu, G. Multifunctional nanostructured conductive polymer gels: synthesis, properties, and applications. Accounts of Chemical Research 2017, 50, 1734-1743.
(3) Chen, W.; Lei, T.; Wu, C.; Deng, M.; Gong, C.; Hu, K.; Ma, Y.; Dai, L.; Lv, W.; He, W.; Liu, X.; Xiong, J.; Yan, C. Designing Safe Electrolyte Systems for a HighStability Lithium-Sulfur Battery. Advanced Energy Materials 2018, 8, 1702348.

(4) Snook, G. A.; Kao, P.; Best, A. S. Conducting-polymer-based supercapacitor devices and electrodes. Journal of Power Sources 2011, 196, 1-12.

(5) van de Burgt, Y.; Lubberman, E.; Fuller, E. J.; Keene, S. T.; Faria, G. C.; Agarwal, S.; Marinella, M. J.; Talin, A. A.; Salleo, A. A non-volatile organic electrochemical device as a low-voltage artificial synapse for neuromorphic computing. Nature Materials 2017, 16, 414-418.

(6) Sunarso, J.; Baumann, S.; Serra, J.; Meulenberg, W.; Liu, S.; Lin, Y.; Da Costa, J. D. Mixed ionic-electronic conducting (MIEC) ceramic-based membranes for oxygen separation. Journal of Membrane Science 2008, 320, 13-41.

(7) Rivnay, J.; Inal, S.; Salleo, A.; Owens, R. M.; Berggren, M.; Malliaras, G. G. Organic electrochemical transistors. Nature Reviews Materials 2018, 3, 1-14.

(8) Wang, H.; Barrett, M.; Duane, B.; Gu, J.; Zenhausern, F. Materials and processing of polymer-based electrochromic devices. $\mathrm{Ma}$ terials Science and Engineering B 2018, 228, 167-174.

(9) Chung, J.; Khot, A.; Savoie, B. M.; Boudouris, B. W. 100th Anniversary of Macromolecular Science Viewpoint: Recent Advances and Opportunities for Mixed Ion and Charge Conducting Polymers. ACS Macro Letters 2020, 9, 646-655.

(10) Rivnay, J.; Inal, S.; Collins, B. A.; Sessolo, M.; Stavrinidou, E.; Strakosas, X.; Tassone, C.; Delongchamp, D. M.; Malliaras, G. G. Structural control of mixed ionic and electronic transport in conducting polymers. Nature Communications 2016, 7, 1-9. 
(11) Smela, E.; Gadegaard, N. Surprising volume change in PPy (DBS): an atomic force microscopy study. Advanced Materials 1999, 11, 953-957.

(12) Thelen, J. L.; Wu, S.-L.; Javier, A. E.; Srinivasan, V.; Balsara, N. P.; Patel, S. N. Relationship between mobility and lattice strain in electrochemically doped poly (3hexylthiophene). ACS Macro Letters 2015, 4, 1386-1391.

(13) Thomas, E. M.; Brady, M. A.; Nakayama, H.; Popere, B. C.; Segalman, R. A.; Chabinyc, M. L. X-Ray Scattering Reveals Ion-Induced Microstructural Changes During Electrochemical Gating of Poly (3-Hexylthiophene). Advanced Functional Materials 2018, 28, 1803687.

(14) Giovannitti, A.; Sbircea, D.-T.; Inal, S.; Nielsen, C. B.; Bandiello, E.; Hanifi, D. A.; Sessolo, M.; Malliaras, G. G.; McCulloch, I.; Rivnay, J. Controlling the mode of operation of organic transistors through side-chain engineering. Proceedings of the National Academy of Sciences 2016, 113, 1201712022.

(15) Zeglio, E.; Eriksson, J.; Gabrielsson, R.; Solin, N.; Inganäs, O. Highly Stable Conjugated Polyelectrolytes for Water-Based Hybrid Mode Electrochemical Transistors. Advanced Materials 2017, 29, 1605787.

(16) Zeglio, E.; Schmidt, M. M.; Thelakkat, M.; Gabrielsson, R.; Solin, N.; Inganäs, O. Conjugated Polyelectrolyte Blends for Highly Stable Accumulation-Mode Electrochemical Transistors. Chemistry of Materials 2017, 29, 4293-4300.

(17) Giridharagopal, R.; Flagg, L.; Harrison, J.; Ziffer, M.; Onorato, J.; Luscombe, C.; Ginger, D. Electrochemical strain microscopy probes morphology-induced variations in ion uptake and performance in organic electrochemical transistors. Nature Materials 2017, 16, 737-742.
(18) Flagg, L. Q.; Bischak, C. G.; Onorato, J. W.; Rashid, R. B.; Luscombe, C. K.; Ginger, D. S. Polymer crystallinity controls water uptake in glycol side-chain polymer organic electrochemical transistors. Journal of the American Chemical Society 2019, 141, 4345-4354.

(19) Kim, S.-M.; Kim, C.-H.; Kim, Y.; Kim, N.; Lee, W.-J.; Lee, E.-H.; Kim, D.; Park, S.; Lee, K.; Rivnay, J.; Yoon, M.-H. Influence of PEDOT:PSS crystallinity and composition on electrochemical transistor performance and long-term stability. Nature Communications 2018, 9, 3858.

(20) Onorato, J. W.; Luscombe, C. K. Morphological effects on polymeric mixed ionic/electronic conductors. Molecular Systems Design \& Engineering 2019, 4, 310324.

(21) Savva, A.; Wustoni, S.; Inal, S. Ionic-toelectronic coupling efficiency in PEDOT: PSS films operated in aqueous electrolytes. Journal of Materials Chemistry C 2018, 6, 12023-12030.

(22) Kunugi, Y.; Harima, Y.; Yamashita, K.; Ohta, N.; Ito, S. Charge transport in a regioregular poly (3-octylthiophene) film. Journal of Materials Chemistry 2000, 10, 2673-2677.

(23) Wang, S.; Ha, M.; Manno, M.; Frisbie, C. D.; Leighton, C. Hopping transport and the Hall effect near the insulator-metal transition in electrochemically gated poly (3hexylthiophene) transistors. Nature Communications 2012, 3, 1-7.

(24) Naoi, K.; Lien, M.; Smyrl, W. H. Quartz crystal microbalance study: ionic motion across conducting polymers. Journal of the Electrochemical Society 1991, 138, 440445.

(25) Qiu, Y.-J.; Reynolds, J. R. Dopant anion controlled ion transport behavior of polypyrrole. Polymer Engineering \& Science 1991, 31, 417-421. 
(26) Nelson, J.; Chandler, R. E. Random walk models of charge transfer and transport in dye sensitized systems. Coordination Chemistry Reviews 2004, 248, 1181-1194.

(27) Modarresi, M.; Franco-Gonzalez, J. F.; Zozoulenko, I. Morphology and ion diffusion in PEDOT: Tos. A coarse grained molecular dynamics simulation. Physical Chemistry Chemical Physics 2018, 20, 17188-17198.

(28) Modarresi, M.; Franco-Gonzalez, J. F.; Zozoulenko, I. Computational microscopy study of the granular structure and $\mathrm{pH}$ dependence of PEDOT: PSS. Physical Chemistry Chemical Physics 2019, 21, 6699-6711.

(29) Mehandzhiyski, A. Y.; Zozoulenko, I. Computational microscopy of pedot: pss/cellulose composite paper. ACS Applied Energy Materials 2019, 2, 3568-3577.

(30) Rolland, N.; Modarresi, M.; FrancoGonzalez, J. F.; Zozoulenko, I. Large scale mobility calculations in PEDOT (Poly (3, 4-ethylenedioxythiophene)): Backmapping the coarse-grained MARTINI morphology. Computational Materials Science 2020, 179, 109678.

(31) Modarresi, M.; Mehandzhiyski, A.; Fahlman, M.; Tybrandt, K.; Zozoulenko, I. Microscopic Understanding of the Granular Structure and the Swelling of PEDOT: PSS. Macromolecules 2020, 53, 6267-6278.

(32) Berardi, R.; Fava, C.; Zannoni, C. A GayBerne potential for dissimilar biaxial particles. Chemical Physics Letters 1998, 297, 814.

(33) Bowen, A. S.; Jackson, N. E.; Reid, D. R.; de Pablo, J. J. Structural correlations and percolation in twisted perylene diimides using a simple anisotropic coarse-grained model. Journal of Chemical Theory and Computation 2018, 14, 6495-6504.

(34) Grozema, F. C.; Van Duijnen, P. T.; Berlin, Y. A.; Ratner, M. A.; Siebbeles, L. D. Intramolecular charge transport along isolated chains of conjugated polymers: effect of torsional disorder and polymerization defects. The Journal of Physical Chemistry $B$ 2002, 106, 7791-7795.

(35) Yesylevskyy, S. O.; Schäfer, L. V.; Sengupta, D.; Marrink, S. J. Polarizable water model for the coarse-grained MARTINI force field. Plos Computational Biology 2010, 6 .

(36) Hutchison, G. R.; Ratner, M. A.; Marks, T. J. Intermolecular charge transfer between heterocyclic oligomers. Effects of heteroatom and molecular packing on hopping transport in organic semiconductors. Journal of the American Chemical Society 2005, 127, 16866-16881.

(37) Almutairi, A. Investigation of sexithiophene properties with Monte Carlo simulations of a coarse-grained model. Ph.D. thesis, University of Akron, 2016.

(38) Rossi, G.; Fuchs, P.; Barnoud, J.; Monticelli, L. A coarse-grained MARTINI model of polyethylene glycol and of polyoxyethylene alkyl ether surfactants. The Journal of Physical Chemistry B 2012, 116, 1435314362.

(39) Franco-Gonzalez, J. F.; Zozoulenko, I. V. Molecular dynamics study of morphology of doped PEDOT: from solution to dry phase. The Journal of Physical Chemistry B 2017, 121, 4299-4307.

(40) Plimpton, S. Fast parallel algorithms for short-range molecular dynamics. Journal of Computational Physics 1995, 117, 1-19.

(41) Cerutti, D. S.; Duke, R. E.; Darden, T. A.; Lybrand, T. P. Staggered mesh Ewald: an extension of the smooth particle-mesh Ewald method adding great versatility. Journal of Chemical Theory and Computation 2009, 5, 2322-2338.

(42) Neelov, A.; Holm, C. Interlaced P3M algorithm with analytical and ik-differentiation. The Journal of Chemical Physics 2010, 132, 234103. 
(43) Hockney, R. W.; Eastwood, J. W. Computer simulation using particles; Bristol: Hilger, 1988, 1988.

(44) Martyna, G. J.; Tobias, D. J.; Klein, M. L. Constant pressure molecular dynamics algorithms. The Journal of Chemical Physics 1994, 101, 4177-4189.

(45) W. M. Brown, S. J. P., M. K. Petersen; Grest, G. S. Liquid crystal nanodroplets in solution. The Journal of Chemical Physics 2009, 130, 044901.

(46) Ryckaert, J.-P.; Ciccotti, G.; Berendsen, H. J. Numerical integration of the cartesian equations of motion of a system with constraints: molecular dynamics of n-alkanes. Journal of Computational Physics 1977, 23, 327-341.

(47) Marcus, R. A. Electron transfer reactions in chemistry. Theory and experiment. Reviews of Modern Physics 1993, 65, 599.

(48) Groves, C. Simulating charge transport in organic semiconductors and devices: a review. Reports on Progress in Physics 2016, 80, 026502.

(49) Brédas, J.-L.; Beljonne, D.; Coropceanu, V.; Cornil, J. Charge-transfer and energytransfer processes in $\pi$-conjugated oligomers and polymers: a molecular picture. Chemical Reviews 2004, 104, 4971-5004.

(50) Poelking, C.; Daoulas, K.; Troisi, A.; Andrienko, D. P3HT Revisited-From Molecular Scale to Solar Cell Devices; Springer, 2014; pp 139-180.

(51) Rühle, V.; Lukyanov, A.; May, F.; Schrader, M.; Vehoff, T.; Kirkpatrick, J.; Baumeier, B.; Andrienko, D. Microscopic simulations of charge transport in disordered organic semiconductors. Journal of Chemical Theory and Computation 2011, 7, 3335-3345.

(52) Barbara, P. F.; Meyer, T. J.; Ratner, M. A. Contemporary issues in electron transfer research. The Journal of Physical Chemistry 1996, 100, 13148-13168.
(53) Rühle, V.; Kirkpatrick, J.; Andrienko, D. A multiscale description of charge transport in conjugated oligomers. The Journal of Chemical Physics 2010, 132, 134103.

(54) Bixon, M.; Jortner, J. Electron transfer-from isolated molecules to biomolecules. Advances in Chemical Physics: Electron Transfer-From Isolated Molecules to Biomolecules. Part 1 1999, 106, 35-202.

(55) Marcus, R. A.; Sutin, N. Electron transfers in chemistry and biology. Biochimica et Biophysica Acta (Bba)-Reviews on Bioenergetics 1985, 811, 265-322.

(56) Marcus, R. A. Chemical and electrochemical electron-transfer theory. Annual Review of Physical Chemistry 1964, 15, 155-196.

(57) Webb, M. A.; Savoie, B. M.; Wang, Z.-G.; Miller III, T. F. Chemically specific dynamic bond percolation model for ion transport in polymer electrolytes. Macromolecules 2015, 48, 7346-7358.

(58) Bello, L.; Sing, C. E. Mechanisms of Diffusive Charge Transport in Redox-Active Polymer Solutions. Macromolecules 2020, 53, 7658-7671.

(59) Cendra, C.; Giovannitti, A.; Savva, A.; Venkatraman, V.; McCulloch, I.; Salleo, A.; Inal, S.; Rivnay, J. Role of the anion on the transport and structure of organic mixed conductors. Advanced Functional Materials 2019, 29, 1807034.

(60) Savva, A.; Cendra, C.; Giugni, A.; Torre, B.; Surgailis, J.; Ohayon, D.; Giovannitti, A.; McCulloch, I.; Di Fabrizio, E.; Salleo, A.; Rivnay, J.; Inal, S. Influence of water on the performance of organic electrochemical transistors. Chemistry of Materials 2019, 31, 927-937.

(61) Moser, M. et al. Side Chain Redistribution as a Strategy to Boost Organic Electrochemical Transistor Performance and Stability. Advanced Materials 2020, 32, 2002748. 
(62) Kaphle, V.; Paudel, P. R.; Dahal, D.; Krishnan, R. K. R.; Lüssem, B. Finding the equilibrium of organic electrochemical transistors. Nature Communications 2020, 11, 111.

(63) Savva, A.; Hallani, R.; Cendra, C.; Surgailis, J.; Hidalgo, T. C.; Wustoni, S.; Sheelamanthula, R.; Chen, X.; Kirkus, M.; Giovannitti, A.; Salleo, A.; McCulloch, I.; Inal, S. Balancing Ionic and Electronic Conduction for High-Performance Organic Electrochemical Transistors. Advanced Functional Materials 2020, 30, 1907657.

(64) Marrink, S. J.; De Vries, A. H.; Mark, A. E. Coarse grained model for semiquantitative lipid simulations. The Journal of Physical Chemistry B 2004, 108, 750-760.

(65) Savagian, L. R.; Österholm, A. M.; Ponder Jr, J. F.; Barth, K. J.; Rivnay, J.; Reynolds, J. R. Balancing charge storage and mobility in an Oligo (Ether) functionalized dioxythiophene copolymer for organic-and aqueous-based electrochemical devices and transistors. Advanced Materials 2018, 30, 1804647.

(66) Dong, B. X.; Nowak, C.; Onorato, J. W.; Strzalka, J.; Escobedo, F. A.; Luscombe, C. K.; Nealey, P. F.; Patel, S. N. Influence of side-chain chemistry on structure and ionic conduction characteristics of polythiophene derivatives: a computational and experimental study. Chemistry of Materials 2019, 31, 1418-1429. 